\title{
Tubular Regeneration: When Can the Kidney Regenerate from Injury and What Turns Failure into Success?
}

\author{
Martin E. Johansson \\ Center for Molecular Pathology, Department of Laboratory Medicine, Lund University, Skåne University Hospital, \\ Malmö, Sweden
}

\section{Key Words}

Kidney regeneration - Acute kidney injury - Tubular damage

\begin{abstract}
Background: The most common intrarenal cause for acute kidney injury/renal failure is tubular damage. The kidney tubules are arranged as compartments of cellular mosaics to perform their functions, and at rest almost a fifth of the human ATP consumption is allotted to the reabsorption of substances from the filtrate, rendering especially the proximal tubules highly sensitive to oxygen and/or nutrient deprivation. Normally mitotically quiescent, the tubular epithelium shows a brisk regenerative response following injury if supportive care is offered, allowing functional restoration. Despite this, the cellular machinery behind the regenerative capacity is still not unequivocally defined. This is at odds with other epithelia such as those of the skin and intestine, where stem cells maintain a continuous flow of new cells from designated niches. Summary: This review discusses the classical concept of renal regeneration, i.e. stochastically surviving cells undergoing dedifferentiation (or epithelial-mesenchymal transition) followed by replenishment of the tubular epithelium. Furthermore however, this view has recently been challenged by the concept of organ-confined stem/progenitor cells, bone marrow-derived stem cells, or mesenchymal
\end{abstract}

stem cells taking part in the regenerative events. Whereas results from animal models support the classical view, morphologically distinct cells have been demonstrated in human kidneys, requiring interpretation. This review presents some of the previous work and techniques and highlights issues that need to be reconciled. Key Messages: In adult humans, the kidney tubules contain scattered cells with a distinct set of markers and properties, such as increased robustness during tubular damage. These cells may be induced by injury or represent a resident progenitor cell pool. To date, animal studies using lineage-tracing methods argue for an inductive scenario. In humans, the situation is less clear and one might speculate that the cellular heterogeneity might reflect elements of cellular reprogramming to a progenitor-like state, perhaps by induction. Due to intense investigational efforts, however, a scientific consensus may soon be reached, which will benefit further research.

(c) 2014 S. Karger AG, Basel

\section{Introduction}

The term acute kidney injury (AKI) is slowly replacing the less distinct former designation, acute renal failure. The condition denotes a clinical syndrome where the glomerular filtration rate is in acute decline, causing a rise in

\section{KARGER}

E-Mail karger@karger.com

www.karger.com/nee (c) 2014 S. Karger AG, Base

$1660-2129 / 14 / 1262-0076 \$ 39.50 / 0$
Martin E. Johansson

Center for Molecular Pathology, Department of Laboratory Medicine, Lund University Skåne University Hospital, Jan Waldenströms gata 59 SE-205 02 Malmö (Sweden)

E-Mail martin.johansson@med.lu.se 

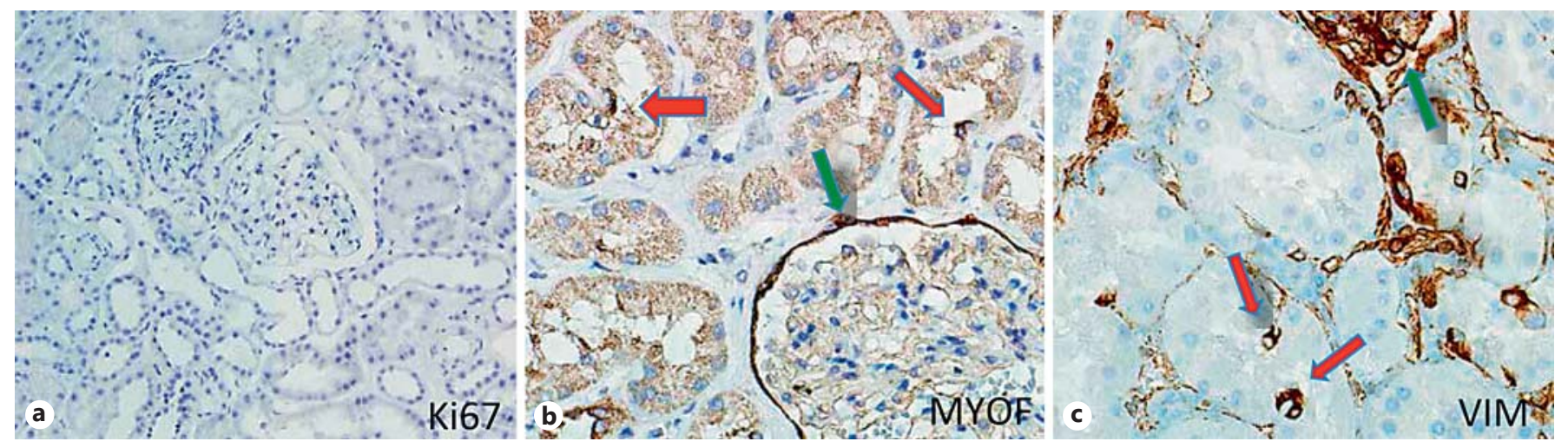

Fig. 1. a Normal human kidney stained for Ki67. No nuclei were positive, and thus no signs of mitotic activity could be seen. $\mathbf{b}$, c Two markers for the scattered cells in the PT. Both were identified by gene expression analyses of ALDEFLUOR-sorted cells. Whereas MYOF is a novel marker for these cells (b), vimentin (VIM) is clas- sically associated with proliferating tubular epithelia (c). Red arrows show the tubular cells and green arrows show the parietal epithelial cells of Bowman's capsule. Interestingly, most markers for the scattered cell population in the PT are indeed also identified in the parietal epithelial cells. Counterstain: hematoxylin. creatinine and, if progressive, the gradual development of uremia. Despite some therapeutic achievements, AKI is still associated with considerable mortality [1]. Of the intrarenal causes for AKI, the overwhelming majority (80$90 \%)$ of cases are due to either ischemia or nephrotoxicity or both $[2,3]$. The main tissue targets for AKI are the renal tubules, and the corresponding pathological changes are called acute tubular necrosis (ATN). This term is overly narrow, however, since the histological changes do encompass cell necrosis but also apoptosis and sloughing of cellular parts or indeed whole viable cells into the tubular lumen. In humans, actual cell necrosis is relatively rarely seen, as opposed to animal model systems for ATN where necrosis dominates the histological changes. Clinically, after the initiation phase, ATN enters the maintenance phase and, if supportive care is offered, a recovery phase follows where the renal tissue (most importantly the tubules) regenerates and kidney function is reestablished.

\section{Tubular Injury}

In health, the renal tubular epithelium is mitotically almost quiescent, in stark contrast to rapidly dividing epithelia such as in the small intestine, skin, or colon. The constant regeneration in these epithelia is driven by stem cells residing in clearly defined niches, but the epithelia of parenchymatous organs do not share these cellular kinetics (fig. 1a). Upon injury, however, the kidneys display a forceful regenerative capacity. AKI is seldom an indication for renal biopsy; therefore, human histological data regarding the distribution of injury along the nephron is limited. Instead, animal experimentation has shown that in general the proximal tubules (PT) are most sensitive to injury, followed by the medullary thick ascending limb part of the distal tubules (DT) [4]. Anatomically, the outer stripe of the outer medulla is most severely affected, and this is most probably due to the relatively low oxygen tension of the medullary region, which is constitutively hypoxic [5]. This environment of low oxygenation continues into the cortex by the medullary ray structures, supplied by venous blood from the medullary region. The medullary rays lack glomeruli, instead harboring the pars recta segments (S3) of the PT and the medullary thick ascending limb, descending into the outer medulla.

Tubular cellular injury may be divided into reversible and irreversible processes. Reversible renal injury results in depolarization of the tubular cell, where normally compartmentalized proteins are redistributed in injured cells [6]. Important examples are the redistribution of integrins and $\mathrm{Na}^{+} / \mathrm{K}^{+}$ATPase to the apical membrane. Irreversible tubular injury results in either apoptosis or necrosis. Animal models most often employ arrest of the renal circulation for a considerable time (20-30 min), resulting in dramatic histological changes, with extensive necrosis and apoptosis. It may be questioned to what degree this correctly emulates the human counterpart. A model more close to human ATN is the animal model of septic AKI, which also produces relatively mild histological changes [7]. 


\section{Tubular Regeneration}

\section{The Classical View}

The undifferentiated metanephric mesenchyme has exhausted its capacity at birth, and any remaining nephrogenic rest at birth represents an aberration [8]. This holds true in most animals with the exception of fish, who retain their nephrogenic capacity during adulthood. Regarding the cellular source for tubular regeneration in mammals, the classical view is that the stochastically least injured cells sustain a reversible measure of injury, followed by dedifferentiation. The cells flatten out to cover the denuded basal membranes, divide, and repopulate the tubules [6]. Finally, tubular integrity is restored as the cells differentiate and repolarize. This view has been well substantiated in rodent model systems by thymidine incorporation assays as, for example, performed by Vogetseder et al. [9], who specifically investigated the $\mathrm{S} 3$ segment of the PT. It has been suggested that tubular cells of this segment are arrested in the G1 phase via cyclin D1, allowing for a tubular cell population that may rapidly respond to tubular injury. More recently a series of studies employed fate-mapping techniques. Cells derived from the Six2-expressing cap mesenchyme formed from the metanephric mesenchyme were used to tag the tubular epithelium [10]. Infliction of ligation-induced injury showed that only cells intrinsic to the tubules took part in the repair. This does not rule out a tubular progenitor cell category, but in a subsequent study, using an unbiased DNA analog-based serial label retention technique, it was demonstrated that regeneration occurred in a random fashion not consistent with a designated progenitor cell population [11]. Recently the proximal tubular epithelium was labeled using a CreERT2 cassette knocked into the locus for SLC34A1. It was shown that the bulk epithelium drives regeneration and a distinct subset of progenitor cells could not be identified. Furthermore, the regenerating cells displayed CD24 and CD133 along with KIM1, markers ascribed to renal progenitors, indicating that these epitopes are markers for dedifferentiation/injury rather than progenitor markers [12]. As authoritative as these studies are, it is important to bear in mind that they all are derived from rodent systems and do not formally establish the situation in the human kidney. Recently, however, it was shown in a rat model of unilateral ureteral obstruction that CD24+/CD133+/vimentin+ cells seem to be inducible during renal injury [13]. That study also showed that the majority of proliferating cells in human regenerating kidneys were CD24 positive.
Recent Ideas on Tubular Regeneration: The Stem/ Progenitor Concept

During the last decade, stem or progenitor cells have been implicated in renal tubular repair. Reports have suggested 3 possible sources: bone marrow-derived stem cells, mesenchymal stem cells, and kidney-confined tubular stem cells [14]. Extrarenal stem cells initially received much interest and these were thought to directly support tubular regeneration by integration into the tubules. Fatemapping experiments have shown conclusively however, that these cells only contribute indirectly to tubular regeneration. The consensus is that they provide soluble factors that support regeneration. This is underscored by the fact that nonrenal cells derived from granulation tissue ameliorate ischemic renal injury (IRI)-induced AKI by endocrine action [15].

Intrarenal stem or progenitor cells have been identified using two major approaches. Either unique properties ascribed to stem cells have been used to isolate this cell population or previously described markers from other stem cell systems have been used to search for kidney stem cells.

\section{Functional Studies}

Renal progenitor cells have been identified in rats using label retention after BrdU staining, where proliferating cells are labeled during the S-phase followed by a washout phase [16]. The method aims at labeling slowly dividing stem cells. The method is based as much on a vigorous washout phase as it is on a labeling phase and, though it is a classical approach in rapidly dividing epithelia, it is unclear to what extent this method may be used in mitotically quiescent parenchymatous organs such as the kidney or liver. Regardless, these cells have been localized to the renal papilla as well as to the renal tubules and it has been shown that the label-retaining cells of the PT account for the majority of cell divisions after renal injury [17]. Label-retaining cells in the renal papillary region have more recently been investigated during searches for mTERT-positive progenitor cells. Genetically labeled mTERT-expressing papillary cells could not be shown to be renal progenitor cells [18].

Side population studies using extrusion of the fluorescent marker Hoechst 33342 exploit the fact that stem cells extrude potentially noxious substances via the $\mathrm{ABC}$ transporter system. The dye-excluding population was shown to express CD133, CD44, and PAX2 to a higher extent than the bulk cells. Also they demonstrated a higher proliferative capacity and an enhanced capacity to form spheres. Flow-based cell sorting according to ALDH1 ac-
78

Nephron Exp Nephrol 2014;126:76-81 DOI: $10.1159 / 000360671$
Johansson 
tivity using the ALDEFLUOR system has allowed enrichment of progenitor-like cells [19]. Gene expression analysis followed by histology identified markers such as myoferlin (fig. 1b) and vimentin (fig. 1c) along with CD133, CD24, and CD44, to mention a few. Another approach used the prolonged culture of renal epithelium by serial passage to select for a cell population that was shown to differentiate into renal tubules when injected subcortically and expressed the markers CD90, PAX2, and Oct 4. Finally, a method employing serial ischemic insults to cultivated kidney epithelium utilized the resistance to apoptosis associated with these cells $[20,21]$.

\section{Marker Studies}

CD133 is a protein of unknown function related to stem cells in other organs. In an early study, CD133+ cells were localized to the renal interstitium [22]. Later studies demonstrated a CD133+ progenitor cell population localized to the parietal epithelial cells of Bowman's capsule [23]. Subsequently, CD133+ cells were also found to be scattered throughout the PT [19]. This cell population seems to share the expression of CD133, CD24, and CD44 $[24,25]$. It was also reported that CD106 acts as a differential marker for progenitor cells localized in Bowmans capsule as opposed to tubular origin, since CD106 is only being expressed by cells at the urinary pole of Bowman's capsule. Less is known regarding the regulation of these cells in relation to both activation and stemness maintenance, but they have been shown to possess multilineage potential in vitro. Also, they have an activated antiapoptotic circuitry and integrate into injured kidney tubules upon IRI. With the probable exception of Bowman's capsule, at present no clear-cut niche has been defined for this cell category. Very recently, however, a study pointed to TLR2 as a receptor involved in the activation of these cells. Also miRNA seems to be involved in cellular maintenance, where miRNA 1915 controls the expression of CD133 and PAX2 in these cells $[24,26]$.

\section{Tubular Regeneration at Organ Level}

Tubular regeneration occurs in anatomical proximity to other structures. As pointed out above, the PT and DT are localized in direct proximity to each other as well as to the vasa recta, a circumstance adding anatomical complexity and the potential for cross talk in regeneration. This theme was recently reviewed by Gobe and Johnson [27]. Briefly, the medulla operates under relative hypoxia as compared to the cortex, due to modest perfusion of the medulla, helping to conserve the hyperosmotic conditions maintained by the countercurrent multiplier sys- tem. The latter process also shifts oxygen from the medullary parts of the kidney, increasing hypoxia. In the outer medulla PT and DT run in parallel, and in the cortical labyrinth, CD, DT, and PT are closely appositioned. DT are more resistant to ischemia and oxidative stress. Furthermore, it has been shown that DT and CD synthesize and store potentially renoprotective substances including HGF, EGF, and IGF-1, factors that the PT do not produce. The PT, however, express receptors for these factors, thereby creating the foundation for protective cross talk during renal injury. The supportive action is further facilitated since the medullary thick ascending part of the DT has a greater capacity to shift from oxidative phosphorylation to glycolysis than the PT [27]. Cell injury also shows structural differences, since DT cells more often undergo apoptosis whereas PT cells are more prone to necrosis. IRI has been shown to enhance the expression of antiapoptotic proteins such as $\mathrm{Bcl}-2$ and $\mathrm{Bcl}-\mathrm{Xl}$ in the DT [28].

\section{Tubular Regeneration as a Recapitulation of Nephrogenesis}

It is known that several of the markers expressed during kidney regeneration are common to nephrogenesis. Among these, we find CD133, CD24, vimentin, and Sall1. Retinoic acid is also a critical factor during renal development as well as during renal regeneration [29]. In this context, it is interesting that ALDH1, the enzyme measured by the ALDEFLUOR assay, is involved in retinoic acid activation. The hypothesis that mature epithelial cells reprogram to a more immature and stem cell-like phenotype is attractive. The similarities may, however, also be confounding in the sense that both the renogenic and the regenerative processes share the same processes connected to motility, cell division, and differentiation.

\section{Future Needs and Unsettled Issues}

Are there kidney stem or progenitor cells in the human kidney? The debate has run parallel to the research during the last decade. Obviously the field would benefit from a reconciliation of the issue. The classicist view of epithelial dedifferentiation has benefited from high-quality research using lineage-tracing models, showing us that at least rodents lack designated stem cells. On the other hand, the proximal tubular epithelium has been shown to harbor not only bulk cells but also a structurally distinct cell type that is interpreted as injury-induced but is also strongly associated with kidney regeneration [13]. Per- 
haps the term state cell would be an alternative to stem or progenitor cell since the cells obviously seem to have a different metabolic state encompassing antiapoptosis, sphere-forming capacity, and a low mitochondrial count compared to the bulk epithelium. Dozens of markers are available today for these cells $[13,19,23]$. If the cells are indeed induced by injury, then we need to explain why there seems to be a perfect marker fit with the parietal cells of Bowman's capsule. These cells cannot be regarded as chronically injured cells, and the matching markers point to a common cellular function. Regardless of their origin, another issue pertains to the choice of models (most renal injury models employ relatively acute and strong injury, such as in IRI models or unilateral ureteral obstruction models). We know little about how the tubular regeneration capacity changes during long-standing chronic injury. There are other interesting phenomena with a possible connection to the issue, such as ischemic preconditioning. It is known that the exposure of cells to hypoxic conditions in various organs, including the kidney, protects against subsequent ischemic insults, an ef- fect mediated in part via the hypoxia-inducible factors HIF- $1 \alpha$ and HIF-2 $\alpha$ [30]. It is tempting to suggest that this mechanism may be connected to the scattered cells with progenitor-like properties. The concepts of induced plasticity and induced stem or progenitor cells are areas of intense investigation and maybe further research will prove the renal cellular heterogeneity to be a long soughtafter in vivo counterpart of these phenomena mostly studied in an in vitro setting. This might bring a consensual end to the game of terms on the issue of tubular regeneration.

\section{Acknowledgements}

Dr. Karl Swärd is thankfully acknowledged for reading the manuscript and providing insightful comments and suggestions. This research was supported by the Marianne and Marcus Wallenberg Foundation, the National Association against Kidney Diseases, Regional ALF Funds, Malmö University Hospital Research and Cancer Funds, and the strategic Cancer Research Program Biocare.

\section{References}

1 Coca SG, Yusuf B, Shlipak MG, Garg AX, Parikh CR: Long-term risk of mortality and other adverse outcomes after acute kidney injury: a systematic review and meta-analysis. Am J Kidney Dis 2009;53:961-973.

$\checkmark 2$ Gill N, Nally JV Jr, Fatica RA: Renal failure secondary to acute tubular necrosis: epidemiology, diagnosis, and management. Chest 2005; 128:2847-2863.

3 Liano F, Pascual J: Epidemiology of acute renal failure: a prospective, multicenter, community-based study. Madrid Acute Renal Failure Study Group. Kidney Int 1996;50: 811-818.

4 Lieberthal W, Nigam SK: Acute renal failure. 1. Relative importance of proximal vs. distal tubular injury. Am J Physiol 1998;275:F623F631.

5 Olsen TS, Hansen HE: Ultrastructure of medullary tubules in ischemic acute tubular necrosis and acute interstitial nephritis in man. APMIS 1990;98:1139-1148.

6 Bonventre JV: Dedifferentiation and proliferation of surviving epithelial cells in acute renal failure. J Am Soc Nephrol 2003;14 (suppl 1):S55-S61.

7 Miyaji T, Hu X, Yuen PS, Muramatsu Y, Iyer S, Hewitt SM, Star RA: Ethyl pyruvate decreases sepsis-induced acute renal failure and multiple organ damage in aged mice. Kidney Int 2003;64:1620-1631.
$>8$ Hartman HA, Lai HL, Patterson LT: Cessation of renal morphogenesis in mice. Dev Biol 2007;310:379-387.

-9 Vogetseder A, Palan T, Bacic D, Kaissling B, Le Hir M: Proximal tubular epithelial cells are generated by division of differentiated cells in the healthy kidney. Am J Physiol Cell Physiol 2007;292:C807-C813.

10 Humphreys BD, Valerius MT, Kobayashi A, Mugford JW, Soeung S, Duffield JS, McMahon AP, Bonventre JV: Intrinsic epithelial cells repair the kidney after injury. Cell Stem Cell 2008;2:284-291.

$\checkmark 11$ Humphreys BD, Czerniak S, DiRocco DP, Hasnain W, Cheema R, Bonventre JV: Repair of injured proximal tubule does not involve specialized progenitors. Proc Natl Acad Sci USA 2011;108:9226-9231.

12 Kusaba T, Lalli M, Kramann R, Kobayashi A, Humphreys BD: Differentiated kidney epithelial cells repair injured proximal tubule. Proc Natl Acad Sci USA 2014;111:15271532.

13 Smeets B, Boor P, Dijkman H, Sharma SV, Jirak P, Mooren F, Berger K, Bornemann J, Gelman IH, Floege J, van der Vlag J, Wetzels JF, Moeller MJ: Proximal tubular cells contain a phenotypically distinct, scattered cell population involved in tubular regeneration. J Pathol 2013;229:645-659.

14 Cantley LG: Adult stem cells in the repair of the injured renal tubule. Nat Clin Pract Nephrol 2005;1:22-32.
15 Patel J, Pancholi N, Gudehithlu KP, Sethupathi P, Hart PD, Dunea G, Arruda JA, Singh AK: Stem cells from foreign body granulation tissue accelerate recovery from acute kidney injury. Nephrol Dial Transplant 2012;27: 1780-1786.

16 Benigni A, Morigi M, Remuzzi G: Kidney regeneration. Lancet 2010;375:1310-1317.

17 Oliver JA, Maarouf O, Cheema FH, Martens TP, Al-Awqati Q: The renal papilla is a niche for adult kidney stem cells. J Clin Invest 2004; 114:795-804.

18 Song J, Czerniak S, Wang T, Ying W, Carlone DL, Breault DT, Humphreys BD: Characterization and fate of telomerase-expressing epithelia during kidney repair. J Am Soc Nephrol 2011;22:2256-2265.

19 Lindgren D, Bostrom AK, Nilsson K, Hansson J, Sjolund J, Moller C, Jirstrom K, Nilsson E, Landberg G, Axelson H, Johansson ME: Isolation and characterization of progenitorlike cells from human renal proximal tubules. Am J Pathol 2011;178:828-837.

20 Gupta S, Verfaillie C, Chmielewski D, Kren S, Eidman K, Connaire J, Heremans Y, Lund T, Blackstad M, Jiang Y, Luttun A, Rosenberg ME: Isolation and characterization of kidneyderived stem cells. J Am Soc Nephrol 2006;17: 3028-3040.

21 Brooks C, Wang J, Yang T, Dong Z: Characterization of cell clones isolated from hypoxia-selected renal proximal tubular cells. Am J Physiol Renal Physiol 2007;292:F243-F252. 
22 Bussolati B, Bruno S, Grange C, Buttiglieri S, Deregibus MC, Cantino D, Camussi G: Isolation of renal progenitor cells from adult human kidney. Am J Pathol 2005;166:545-555.

-23 Sagrinati C, Netti GS, Mazzinghi B, Lazzeri E, Liotta F, Frosali F, Ronconi E, Meini C, Gacci M, Squecco R, Carini M, Gesualdo L, Francini F, Maggi E, Annunziato F, Lasagni L, Serio M, Romagnani S, Romagnani P: Isolation and characterization of multipotent progenitor cells from the Bowman's capsule of adult human kidneys. J Am Soc Nephrol 2006;17: 2443-2456.

-24 Sallustio F, De Benedictis L, Castellano G, Zaza G, Loverre A, Costantino V, Grandaliano G, Schena FP: TLR2 plays a role in the activation of human resident renal stem/progenitor cells. FASEB J 2010;24:514-525.
25 Angelotti ML, Ronconi E, Ballerini L, Peired A, Mazzinghi B, Sagrinati C, Parente E, Gacci M, Carini M, Rotondi M, Fogo AB, Lazzeri E, Lasagni L, Romagnani P: Characterization of renal progenitors committed toward tubular lineage and their regenerative potential in renal tubular injury. Stem Cells 2012;30:17141725.

26 Sallustio F, Serino G, Costantino V, Curci C, Cox SN, De Palma G, Schena FP: miR-1915 and miR-1225-5p regulate the expression of CD133, PAX2 and TLR2 in adult renal progenitor cells. PLoS One 2013;8:e68296.

27 Gobe GC, Johnson DW: Distal tubular epithelial cells of the kidney: potential support for proximal tubular cell survival after renal injury. Int J Biochem Cell Biol 2007;39:15511561.
28 Cuttle L, Zhang XJ, Endre ZH, Winterford C, Gobe GC: Bcl-X(L) translocation in renal tubular epithelial cells in vitro protects distal cells from oxidative stress. Kidney Int 2001; 59:1779-1788.

29 Cirio MC, de Groh ED, de Caestecker MP, Davidson AJ, Hukriede NA: Kidney regeneration: common themes from the embryo to the adult. Pediatr Nephrol 2014;29:553-564.

30 Bonventre JV: Kidney ischemic preconditioning. Curr Opin Nephrol Hypertens 2002; 11:43-48. 